\title{
Concentración de fluoruros en la sal de uso doméstico comercializada en Montevideo, Uruguay
}

\author{
Concentration of fluorides in household salt \\ marketed in Montevideo, Uruguay
}

Concentração de flúor no sal de uso doméstico comercializado em Montevidéu, Uruguai

\author{
Ana Paula Dall'Onder ${ }^{1}$ (D) 0000-0003-3066-4660 \\ Anunzziatta Fabruccini Fager ${ }^{2}$ (iD 0000-0001-7344-4751 \\ Licet Alvarez Loureiro ${ }^{2}$ (D) 0000-0001-9659-6045 \\ Lina Naomi Hashizume ${ }^{3}$ (D) 0000-0001-5477-2768
}

\section{Resumen}

Objetivos: Evaluar la concentración de fluoruro en la sal de mesa disponible comercialmente en Montevideo, Uruguay.

Método: Fueron analizados catorce paquetes de sal para determinar la concentración de iones fluoruro libres. Las muestras se pesaron, se diluyeron en agua desionizada, se mezclaron con TISAB II y se utilizó un electrodo específico conectado a un analizador de iones para determinar la concentración de fluoruro

Resultado: La mayoría de los paquetes presentaron valores inferiores a $250 \mathrm{mgF} / \mathrm{kg}$. Las muestras de sal gruesa mostraron concentraciones mayores a las de sal fina $(\mathrm{p}<0.05)$. Aquellas conteniendo fluoruro de sodio presentaron mayor concentración de fluoruro que aquellas conteniendo fluoruro de potasio $(\mathrm{p}<0.05)$. Dos marcas presentaron concentraciones de fluoruro cercanas a las informadas en el envase.

Conclusiones: Las sales de mesa comercializadas en Montevideo, Uruguay presentaron gran variabilidad en las concentraciones de fluoruros.

Palabras clave: fluoruros, sal, caries dental, Uruguay.

Departamento de Odontología Preventiva y Social, Facultad de Odontología, UFRGS, Porto Alegre, Brasi

Cátedra de Odontopediatría, Facultad de Odontología, Universidad de la Republica, Montevideo, Uruguay

Departamento de Odontología Preventiva y Socia, Facultad de Odontología, UFRGS, Porto Alegre, Brasil. lhashizume@yahoo.com

Fecha de recibido: 24/2/2021 - Fecha de aceptado: 28/6/2021 


\section{Abstract}

Objectives: The aim of this study was to evaluate the concentration of fluoride (F) in household salt marketed in Montevideo, Uruguay.

Methods: Fourteen household salt brands marketed in Montevideo, Uruguay were analyzed to determine the $\mathrm{F}$ concentrations. Salt samples were prepared and F concentrations were determined using a fluoride-specific electrode connected to an ion analyzer. Results: Most of the salt brands showed values lower than $250 \mathrm{mgF} / \mathrm{kg}$. Coarse salt samples showed higher $F$ concentrations compared to the refined salt samples ( $\mathrm{p}<$ 0.05). Salt brands contained sodium fluoride presented higher $\mathrm{F}$ concentrations than salt brands contained potassium fluoride ( $\mathrm{p}$ $<0.05$ ). Only two brands had F concentrations close to that informed in product packages.

Conclusions: Household salt brands marketed in Montevideo (Uruguay) present a great variability in their $\mathrm{F}$ concentrations.

Keywords: fluoride; salt; dental caries; Uruguay.

\section{Introducción}

El acceso a nivel comunitario del fluoruro, a través del agua y la sal ha demostrado ser una medida de salud pública para la disminución de la caries dental en el mundo ${ }^{(1)}$. La fluoruración de la sal es una medida que ha venido creciendo, principalmente en los países en desarrollo, donde la fluoruración del agua ha demostrado ser inaccesible debido a sistemas de suministro y distribución de agua descentralizados e inadecuados ${ }^{(2-4)}$.

\section{Resumo}

O objetivo deste estudo foi avaliar as concentraçôes de flúor ( $\mathrm{F}$ ) no sal de uso doméstico comercializado em Montevidéu, Uruguai.

Métodos: Quatorze marcas de sal doméstico comercializadas em Montevidéu (Uruguai) foram analisadas para determinar as concentrações de F. As amostras de sal foram preparadas e as concentraçóes de flúor foram determinadas através de um eletrodo específico para $\mathrm{F}$ conectado a um analisador de íons.

Resultados: A maioria das marcas de sal mostraram valores menores que $250 \mathrm{mgF} /$ $\mathrm{kg}$. Amostras de sal grosso apresentaram as maiores concentraçōes de $\mathrm{F}$ comparadas às amostras de sal refinado $(\mathrm{p}<0,05)$. Apenas duas marcas tiveram as concentraçóes de $\mathrm{F}$ próximas àquelas informadas nas embalagens dos produtos.

Conclusôes: As marcas de sal de uso doméstico comercializadas em Montevidéu (Uruguai) apresentam uma grande variabilidade em suas concentrações de $\mathrm{F}$.

Palavras-chave: fluoreto; sal; cárie dentária; Uruguai.

La fluoruración comunitaria de la sal está disponible en Uruguay desde 1991, llegando a casi toda la población ${ }^{(5)}$. En comparación con la fluoruración del agua, esta alternativa demostró una reducción similar en la prevalencia de caries al tiempo que brinda la posibilidad a los consumidores de optar por ella ${ }^{(2)}$. La población uruguaya posee acceso a sal fluorada de uso doméstico en una concentración de $250 \mathrm{mgF} /$ $\mathrm{kg}^{(6)}$.

Los estudios realizados en otros países de América Latina analizaron las concentraciones de 
fluoruro de las sales de mesa y encontraron grandes variaciones entre las marcas de sal ${ }^{(7-12)}$. Además, en la mayoría de las marcas de sal no se correspondía con la concentración de flúor informada en la etiqueta del producto, en desacuerdo con la legislación del país. Sin embargo, no fueron encontrados trabajos que reporten el contenido de fluoruro en la sal comercializada en Uruguay. Por lo tanto, el objetivo del presente estudio fue evaluar las concentraciones de fluoruro de diferentes marcas de sales de mesa disponibles comercialmente en Montevideo, Uruguay.

\section{Metodología}

\section{Muestra}

Un estudio analítico se llevó a cabo para evaluar la concentración de fluoruro en catorce paquetes de sal fluorada disponibles comercialmente en supermercados y tiendas de comestibles ubicados en diferentes zonas de Montevideo, Uruguay. Tres paquetes de la misma marca, con diferente numeración de lote, fueron adquiridos. Las muestras fueron codificadas con diferentes números para permitir un análisis ciego. Todas las muestras fueron analizadas entre Octubre de 2016 y Enero de 2017, antes de su fecha de expiración. La Tabla 1 muestra la información declarada en la etiqueta del producto, aportada por el fabricante.

\section{Análisis de concentración de fluoruro}

Las muestras de sal fueron analizadas para determinar la concentración de iones fluoruro libres. El análisis procedió de la siguiente manera: 0.25 $\mathrm{g}$ de sal fueron diluídos en tubos conteniendo 1 $\mathrm{mL}$ de agua destilada y desionizada. Cada una de las muestras fué mezclada en 1:1 (v/v) con TISAB II (Fisher Scientific Co., USA). Posteriormente, las muestras fueron analizadas con un electrodo específico para fluoruros, conectado a un analizador de iones, de acuerdo a la técnica utilizada por Mier y cols. (2009) ${ }^{(13)}$.
Se realizó un análisis en duplicado de diez muestras, en dos momentos diferentes, con un intervalo de una semana, para determinar la reproducibilidad intra-examinador. Fueron realizados análisis adicionales para intentar explicar las diferencias observadas en los resultados iniciales. Para calibrar el equipo (electrodo Orion 96-09 acoplado a un analizador de iones Orion Star A214; Thermo Scientific, Cambridge, MA, USA) se utilizaron soluciones standard de fluoruro que iban de 0,25 a $16,0 \mu \mathrm{g} \mathrm{F} / \mathrm{ml}$, mezclado con TISAB II (1:1, v/v) sumado a $0,025 \mathrm{~g}$ $\mathrm{NaCl} / \mathrm{mL}$. Cada una de estas se mezclaron en 1:1 (v/v) en un recipiente con TISAB II ${ }^{(13)}$.

\section{Análisis de los datos}

Fue realizada una regresión lineal del logaritmo de las concentraciones de fluoruro de las soluciones estándar y se calcularon los respectivos valores de $\mathrm{mV}$. La ecuación matemática de regresión se utilizó para determinar la concentración de fluoruro de las soluciones salinas (en miligramos de fluoruro). Se calcularon las concentraciones medias y desviaciones estándar para las dos alícuotas de cada envase y para los tres envases de cada marca de sal.

Se utilizó la prueba t-Student para comparar los grupos de sales (gruesa vs fina y $\mathrm{NaF}$ vs $\mathrm{KF}$ ). La significación estadística se fijó en $p<0,05$. Todos los análisis estadísticos se realizaron con el programa SPSS versión 18.0 (SPSS Inc., Chicago, IL). Las concentraciones encontradas en las marcas de sal analizadas se compararon con la información aportada por la etiqueta del envase.

\section{Resultados}

Entre las catorce marcas de sal fluorada disponibles comercialmente en Montevideo, Uruguay, analizadas en el presente estudio, sólo seis marcas fueron producidas en Uruguay (muestras 3, 4, 5, 6, 11 y 12). Las otras ocho marcas de sal fueron importadas de Argentina (muestras 1, 2, 7, 8, 9, 10 y 13) y de Brasil (muestra 14). 
Tabla 1: Información especificada por los fabricantes en los paquetes de sal

\begin{tabular}{|c|c|c|c|c|c|}
\hline $\begin{array}{l}\text { Marca comercial } \\
\text { (Fabricante) País }\end{array}$ & Código & Tipo de sal & $\begin{array}{l}\text { Compuesto } \\
\text { de fluoruro }\end{array}$ & $\begin{array}{l}\text { Concentración de } \\
\text { fluoruro declarada } \\
\qquad(\mathrm{mg} / \mathrm{kg})\end{array}$ & Otros componentes activos \\
\hline $\begin{array}{c}\text { Monte Cudine } \\
\text { (Haidar y cia. SRL) } \\
\text { Argentina }\end{array}$ & 1 & Fina & $\mathrm{NaF}$ & 250 & $\mathrm{NaCl} ;$ Yodato de potasio; INS 341iii \\
\hline $\begin{array}{c}\text { Monte Cudine } \\
\text { (Haidar y cia. SRL) } \\
\text { Argentina }\end{array}$ & 2 & Gruesa & $\mathrm{NaF}$ & 250 & $\mathrm{NaCl} ;$ Yodato de potasio; INS 341iii \\
\hline $\begin{array}{c}\text { Sal Sek } \\
\text { (Deambrosi) Uruguay }\end{array}$ & 3 & Fina & $\mathrm{NaF}$ & 250 & $\begin{array}{l}\text { Yodato de potasio } 30 \mathrm{ppm} / \text { Fosfato tricál- } \\
\text { cico } 0,3 \%\end{array}$ \\
\hline $\begin{array}{c}\text { Sal Sek } \\
\text { (Deambrosi) Uruguay }\end{array}$ & 4 & Gruesa & KF & 250 & $\begin{array}{l}\text { Yodato de potasio } 30 \mathrm{ppm} / \text { Fosfato tricál- } \\
\text { cico } 0,3 \%\end{array}$ \\
\hline $\begin{array}{c}\text { Urusal } \\
\text { (Anti SA) Uruguay }\end{array}$ & 5 & Gruesa & $\mathrm{NaF}$ & 250 & $\mathrm{NaCl}$; Yodato de potasio 30 ppm \\
\hline $\begin{array}{c}\text { Urusal } \\
\text { (Anti SA) Uruguay }\end{array}$ & 6 & Fina & $\mathrm{NaF}$ & 250 & $\mathrm{NaCl}$; Yodato de potasio 30 ppm / 3413iii \\
\hline $\begin{array}{c}\text { Dos Anclas } \\
\text { (Salinas Grandes, } \\
\text { Hidalgo La Pampa) } \\
\text { Argentina }\end{array}$ & 7 & Gruesa & $\begin{array}{c}\text { No } \\
\text { declarado }\end{array}$ & 250 & 30 ppm de yodo \\
\hline $\begin{array}{c}\text { Dos Anclas } \\
\text { (Salinas Grandes, } \\
\text { Hidalgo La Pampa) } \\
\text { Argentina }\end{array}$ & 8 & Fina & $\begin{array}{c}\text { No } \\
\text { declarado }\end{array}$ & 250 & $\begin{array}{l}30 \text { ppm de yodo / dióxido de silicio 1,5\% } \\
\text { INS } 515\end{array}$ \\
\hline $\begin{array}{c}\text { Celusal } \\
\text { (Industrias químicas } \\
\text { y mineras Timbo SA) } \\
\text { Argentina }\end{array}$ & 9 & Gruesa & KF & 250 & $\mathrm{NaCl}$; Yodato de potasio $30 \mathrm{ppm}$ \\
\hline $\begin{array}{c}\text { Celusal } \\
\text { (Industrias químicas } \\
\text { y mineras Timbo SA) } \\
\text { Argentina }\end{array}$ & 10 & Fina & KF & 250 & $\begin{array}{l}\mathrm{NaCl} ; \text { Yodato de potasio } 30 \mathrm{ppm} / \mathrm{INS} \\
\text { 551ioins } 536\end{array}$ \\
\hline $\begin{array}{l}\text { Cololo } \\
\text { (Solsire SA) Uruguay }\end{array}$ & 11 & Gruesa & KF & 250 & Yodato de potasio $30 \mathrm{ppm}$ \\
\hline $\begin{array}{l}\text { Cololo } \\
\text { (Solsire SA) Uruguay }\end{array}$ & 12 & Fina & $\mathrm{NaF}$ & 250 & Yodato de potasio / fosfato tricálcico \\
\hline $\begin{array}{c}\text { Dos Estrellas } \\
\text { (Salinas Grandes, } \\
\text { Hidalgo La Pampa) } \\
\text { Argentina }\end{array}$ & 13 & Gruesa & $\begin{array}{l}\text { No } \\
\text { declara }\end{array}$ & 250 & 30 ppm de yodo \\
\hline $\begin{array}{l}\text { Marina Diamante } \\
\text { (Romani SA) Brasil }\end{array}$ & 14 & Fina & $\begin{array}{l}\text { No } \\
\text { declara }\end{array}$ & 250 & $\begin{array}{l}\mathrm{NaCl} \text {, yodato de potasio, anti-vaho, fosfato } \\
\text { tricálcico, } 30 \mathrm{ppm} \text { de yodo }\end{array}$ \\
\hline
\end{tabular}

$\mathrm{NaF}$ : fluoruro de sodio, KF: fluoruro de potasio 
Los valores medios para la concentración de fluoruro encontrados en cada muestra de sal analizada en este estudio son mostrados en la Figura 1, estos variaron en un rango de 19,22 a 553,42 mgF / kg. La mayoría de las marcas comerciales de sal disponibles en Uruguay presentaron valores de concentración de fluoruro por debajo de $250 \mathrm{mgF} / \mathrm{kg}$. Solo dos marcas de sal (muestras 10 y 14) alcanzaron el promedio esperado de concentración de fluoruro $(250$ $\mathrm{mgF} / \mathrm{kg}$ ) y tres marcas mostraron valores muy altos (muestras 2, 7 y 13). Se verificó una gran diferencia entre los valores de las concentraciones de fluoruro comparando los distintos lotes de las mismas marcas de sal, lo que reflejó una amplia desviación estándar (Figura 1).

Seis de las marcas de sal analizadas estaban disponibles en dos presentaciones diferentes: fina y gruesa. El valor medio de la concentración de flúor en las sales finas fue de 115,56 $\pm 109,09$ $\mathrm{mgF} / \mathrm{kg}$ (media $\pm \mathrm{DE})$, con un Coeficiente de
Variación $(\mathrm{CV})=94,4 \%$. En las sales gruesas, el valor medio de la concentración de flúor fue de $242,33 \pm 128,35 \mathrm{mgF} / \mathrm{kg}$ (media $\pm \mathrm{DE}$ ), con un $\mathrm{CV}=53,0 \%$. En todas las marcas de sal, la sal gruesa presentó valores más altos de concentración de flúor en comparación con la presentación de sal fina de la misma marca $(\mathrm{p}<0,05)$. El fluoruro de sodio $(\mathrm{NaF})$ y el fluoruro de potasio (KF) fueron los compuestos de fluoruro incorporados en las marcas de sal analizadas en el presente estudio. Las marcas de sal que contenían KF (muestras 4, 9, 10 y 11) presentaron mayores concentraciones de flúor en comparación con las marcas de sal que contenían $\mathrm{NaF}$ (muestras 1, 2, 3, 5, 6, 12) ( $\mathrm{p}<0,05)$. La concentración de flúor de las sales que contenían $\mathrm{NaF}$ fue de $125,76 \pm 150,77 \mathrm{mg} \mathrm{F} / \mathrm{kg}$ (media \pm $\mathrm{DE}$ ), con un $\mathrm{CV}=120 \%$, mientras que las marcas de sal con $\mathrm{KF}$ presentaron la concentración de 214,49 \pm 97,89 $\mathrm{mg} \mathrm{F} / \mathrm{kg}$ (media $\pm \mathrm{DE}$ ) con un $\mathrm{CV}=45,6 \%$.

Figura 1: Concentraciones de fluoruro ( $\mathrm{mg} / \mathrm{kg}$ ) encontradas en cada una de las muestras de sal (identificadas por sus número de código) analizadas en este estudio.

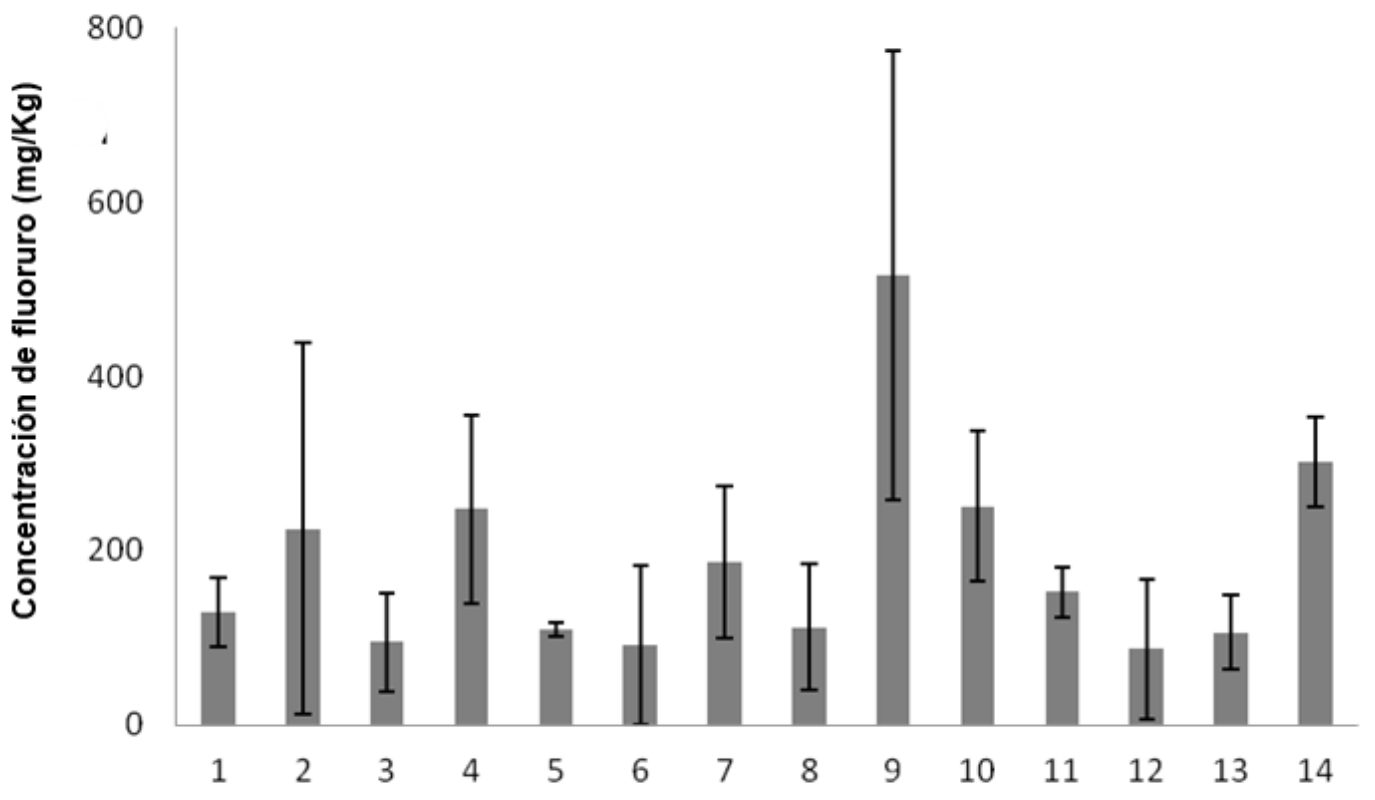

Códigos de las marcas de sal 


\section{Discusión}

En 1991, Uruguay introdujo el Programa Nacional de Fluoruración de la sal de uso doméstico en todo el país, en una concentración de 250 mg F/kg. En muchos países de América Latina fueron fluoradas todas las sales de consumo humano, mientras que en Uruguay sólo se hizo para la de uso doméstico. En consecuencia se ha elaborado una legislación específica, y el gobierno uruguayo exige que el $60 \%$ de la sal para uso doméstico esté yodada y fluorada en todo el país ${ }^{(6)}$.

La elección de un método poblacional de uso de fluoruros aporta beneficios a la población en general, incluyendo personas de estratos socioeconómicos bajos que no tienen acceso a los dentífricos fluorados y a las aplicaciones profesionales. Varios países de América Latina utilizan la sal fluorada como método poblacional de distribución de fluoruro, como Colombia, Costa Rica, México y Jamaica. Uruguay es uno de los países que cubre prácticamente a toda la población ${ }^{(14)}$.

La mayoría de las marcas de sales disponibles en Uruguay, analizadas en el presente estudio, presentaron valores de fluoruro discordante con lo declarado en el envase y lo recomendado por la legislación. Aun cuando la etiqueta anuncia que la sal comercializada tiene $250 \mathrm{mg} \mathrm{F} / \mathrm{kg}$, los valores encontrados en este estudio contradicen esta información. Los valores de concentración de fluoruro de las muestras analizadas iban desde niveles muy bajos, como 19,22 mg F/ kg, hasta niveles muy altos como 553,42 mg F/kg. Solamente dos marcas tenían una media aproximada a la declarada de $250 \mathrm{mg}$ F/ kg (muestras 10 y 14).

Varias marcas de sal mostraron altas desviaciones estándar, que reflejan la gran diferencia de resultados encontrados en los diferentes lotes de la misma marca de sal (Figura 1). Por lo tanto, se puede asumir que no existe un control de calidad adecuado en el proceso de fluoruración de la sal, que estandarice la cantidad correcta a incorporar en todos los lotes fabricados.
Por otro lado, puede sospecharse de una falta de homogeneidad en la incorporación de flúor en las partículas de sal dentro de cada envase. Es posible que el tamaño de las partículas de sal influya en la incorporación de flúor. Al comparar las presentaciones de sales finas y gruesas de la misma marca, las sales gruesas tenían valores más altos de flúor en comparación con las sales finas en todas las muestras analizadas.

El método de fluoruración de la sal también puede influenciar los resultados. Las marcas de sal que fueron fluoradas por el método húmedo (muestras 4, 9, 10 y 11), utilizaron KF como compuesto fluorado y presentaron mayores concentraciones de fluoruro en comparación con las fluoruradas por el método seco utilizando $\mathrm{NaF}$ (muestras 1, 2, 3, 5, 6, 12). Además, las marcas de sal por método húmedo presentaron menos variabilidad de concentración de flúor comparadas con las del método seco.

Dos estudios observacionales ${ }^{(15,16)}$ y un ensayo clínico ${ }^{(18)}$ compararon diferentes métodos de distribución de flúor a nivel poblacional (sal fluorada y agua fluorada). En los estudios de Mejía et al. (1976) ${ }^{(17)}$ y Sagheri et al. (2007) ${ }^{(15)}$ no hubo diferencias entre los distintos métodos de fluoruración, lo que refuerza el importante papel de la sal fluorada en las zonas donde no es posible la fluoruración del agua. El estudio de Mejía et al. (1976) ${ }^{(17)}$ se llevó a cabo en diferentes comunidades colombianas, mientras que el estudio de Sagheri et al. (2007) ${ }^{(15)}$ se realizó en Dublín (agua fluorada) y Friburgo (sal fluorada).

Sin embargo, el estudio realizado por Fabruccini et al. (2016) ${ }^{(16)}$ arrojó conclusiones diferentes a las de los estudios anteriores. Comparó el efecto de la fluoruración del agua en Porto Alegre, Brasil, con la fluoruración de la sal en Montevideo, Uruguay. Sus resultados demostraron que la sal fluorada proporciona menos protección contra la caries dental en los escolares que el agua fluorada, y que los nińos que consumían sal fluorada tenían un CPOD significativamente mayor que los expuestos al agua 
fluorada, independientemente del criterio utilizado (OMS o OMS modificado). Los autores atribuyeron sus resultados al hecho de que el programa de fluoruración de la sal en Uruguay se limita a la sal doméstica, sin cobertura en comedores y restaurantes, lo que puede haber reducido su impacto en la población ${ }^{(16)}$.

Este es el primer estudio que analiza las concentraciones de flúor contenidas en las marcas de sal domestica disponibles en Uruguay. El presente estudio demostró que la mayoría de las marcas de sal fluorada uruguayas tenían concentraciones de flúor inferiores a las recomendadas para ser efectivas contra la caries dental. Este hallazgo también puede ayudar a entender los resultados de Fabruccini et al. (2016) (16) donde el efecto protector de la sal fluorada disponible en Uruguay fue menor en comparación con el del agua fluorada de Brasil. La variabilidad de la concentración de fluoruro respecto a las declaraciones del envase y a las recomendaciones encontradas hace pensar en la necesidad de mejorar la regulación en su proceso de fabricación. La población uruguaya sólo cuenta con este método comunitario de uso de fluoruro como medida de salud pública para prevenir la caries dental. Los resultados muestran que no está siendo beneficiada por este método, debido a que está expuesta a niveles muy bajos o muy altos de fluoruro.

El estudio de Maupomé-Carvantes et al. (1995) (8) también evaluó la concentración de flúor en muestras de sal en la Ciudad de México y encontró resultados similares al presente estudio. De los 221 paquetes de sal analizados por los autores, la mayoría presentaba cantidades de flúor por debajo de la norma gubernamental, ya que el $50,2 \%$ de las muestras tenían de 1 a $50 \mathrm{mg} \mathrm{F} / \mathrm{kg}$ de flúor, en desacuerdo con lo anunciado en los paquetes (250 mg F/ kg).

Con el fin de que la fluoruración de la sal en Uruguay sea efectiva a nivel poblacional y presente mejores resultados en el control de la caries dental en los índices epidemiológicos a lo largo de los años, se requiere una mejora en el proceso de fabricación de la sal, con un mayor control durante la fluoruración de la sal de mesa, incluso para que todas las marcas puedan presentar el valor recomendado de flúor independientemente de los lotes fabricados, el tipo de fluoruración y el tamaño de las partículas de la sal, de forma que se pueda ofrecer un producto de calidad al consumidor.

Los resultados se basan en el análisis de productos comercializados en el año 2016, igualmente consideramos vigentes los mismos debido a la ausencia de cambios en los mecanismos de vigilancia del Programa Nacional de Fluoruración de la Sal.

\section{Conclusión}

Hay una gran variabilidad en las concentraciones de flúor en las marcas de sal de mesa fluorada disponibles comercialmente en Montevideo, Uruguay. La mayoría de las marcas de sal analizadas no se corresponden con la información mencionada en la etiqueta del producto, presentando desde resultados muy bajos hasta resultados muy superiores a los esperados. Es posible que la población de Uruguay no esté recibiendo los beneficios esperados por este método debido a la falta de control de calidad, por lo que se requiere una mayor regulación del proceso de fabricación de la sal de mesa fluorada.

\section{Referencias}

1. Tenuta LMA, Cury JA. Fluoride: its role in dentistry. Braz Oral Res. 2010; 24 (Suppl 1):9 -17.

2. Estupinán-Day S. Promoting oral health: the use of salt fluoridation to prevent dental caries. Washington: PAHO, 2005. 
3. Horowitz HS. Decision-making for national programs of community fluoride use. Community Dent Oral Epidemiol. 2000; 28(5):321-9.

4. Marthaler T, Petersen P. Salt fluoridation- an alternative in automatic prevention of dental caries. Int Dent J. 2005;55(6):351-8.

5. Ditterich RG, Rodrigues CK, Wambier DS. [Fluoride salt as a alternative in public oral health: advantages and disadvantages]. Rev Inst Ciênc Saúde, 2005; 23(3): 231-4.

6. Comisión Honoraria de Salud Bucal. Ministerio de Salud Pública (MSP) Encuesta de Salud Bucal en escolares de 11 a 14 ańos. Sector Público. República Oriental del Uruguay. 1999.

7. Martínez-Mier EA, Soto Rojas AE, Buckley CM, Stookey GK, Zero DT, Margineda J. Evaluación del contenido de flúor en la sal de mesa fluorada. Salud Pública Mex. 2004 May-Jun; 46(3):2003-4.

8. Walsh K I and Cury J A. Fluoride concentrations in salt marketed in Managua, Braz. Oral Res. 2018;32: e45

9. Hernández-Guerrero JC, Fuente-Hernández J, Jiménez-Farfán MD, Ledesma-Montes C, CastañedaCastaneira E, Molina-Frechero N, et al. Fluoride content in table salt distributed in Mexico City, Mexico. J Public Health Dent. 2008; 68(4):242-5.

10. Franco AM, Saldarriaga A, Gonzalez MC, Martignon S, Arbelaez MI, Ocampo A. Concentración de fluor en la sal de cocina en cuatro ciudades colombianas. Revista CES Odontología 2003;16: 21-6.

11. Tovar Valencia S, Castrillon Misnaza S. Perspectiva del uso del flúor vs caries y fluorosis dental. Colombia: Minsalud; 2016 [cited 2017 Sep 29] Disponible en: http://www.minsalud.gov.co/sites/rid/Lists/ Bibliotecadigital/RIDE//VS/PP/ENT/perspectiva-uso-fluor.pdf.

12. Walsh KI, Cury JA. Fluoride concentrations in salt marketed in Managua, Nicaragua. Braz Oral Res. 2018; 24, 32:e45.

13. Mier EAM, Rojas AES, Buckley CM, Margineda J, Zero DT. Evaluation of the direct and diffusion methods for the determination of fluoride content in table salt. Community Dent Health, 2009; 26(4): 204-10.

14. Marthaler TM. Salt Fluoridation and Oral Health. Acta Medica Academica, 2013; 42(2): 140-55.

15. Sagheri D, Mcloughlin J, Clarckson J J. A comparison of dental caries levels in two communities with different oral health prevention strategies stratified in different social classes. Journal of Public Health Dentistry, 2007; 67(1): 1-7.

16. Fabruccini A, Alves LS, Alvarez L, Alvarez R, Susin C, Maltz M. Comparative Effectiveness Of Water And Salt Community-Based Fluoridation Methods In Preventing Dental Caries Among Schoolchildren. Community Dent. Oral Epidemiol, 2016; 44(6): 577-85.

17. Mejía R, Espinal F, Vélez H, Aguirre M. Fluoruración de la sal en cuatro comunidades colombianas. VIII. Resultados obtenidos de 1964 a 1972. Bol Oficina Sanit Panam. Colombia, 1976; 80: 205.

\section{Nota conflicto de interés:}

Los autores declaran no tener ningún conflicto de intereses en la realización de este trabajo y el reporte de sus resultados. 